\title{
The Wanderer: Um jogo para auxiliar no combate ao sedentarismo e promover uma vida saudável
}

\author{
Raphael Felipe do Vale Lucena ${ }^{1}$, Francisco das C. Imperes Filho ${ }^{2}$, \\ Alan Rafael F. dos Santos ${ }^{2}$, Leonardo Pereira de Sousa ${ }^{2}$ \\ ${ }^{1}$ Universidade Federal do Piuí (UFPI) - Campus Senador Helvídio Nunes de \\ Barros (CNHNB) - CEP: 64.607-670 - Picos - PI - Brazil \\ ${ }^{2}$ Núcleo de Computação de Alto Desempenho - Universidade Federal do Piú́ (UFPI) \\ CEP: 64.049-550 - Teresina - PI - Brazil \\ raphaelfelipe999@gmail.com, \{fcomimperes, leonardosousa\}@ufpi.edu.br \\ alan_rafael118@hotmail.com
}

\begin{abstract}
The present work describes the usability tests and development of a mobile game, The Wanderer. Its main objective is to encourage and keep the player engaged in the practice of physical activities, such as walking and running. Usability tests were applied using an online questionnaire. The work obtained good results in the software usability tests, obtaining an average of 90,6 in the SUS method and an average acceptance percentage of 94,62\%. The game proved to be promising in its proposal and efficient in taking the user to practice physical activities.
\end{abstract}

Resumo. O presente trabalho descreve os testes de usabilidade e desenvolvimento de um jogo para celular, The Wanderer. Seu principal objetivo é incentivar e manter o jogador engajado na prática de atividades físicas, como caminhada e corrida. Os testes de usabilidade foram aplicados utilizando questionário online. O trabalho obteve bons resultados nos testes de usabilidade do software, obtendo uma média de 90,6 no método SUS e um percentual de aceitação médio de 94,62\%. O jogo mostrou-se promissor em sua proposta e eficiente em levar o usuário a prática de atividades físicas.

\section{Introdução}

O sedentarismo é a falta ou ausência de atividades físicas, levando a uma redução nos gastos calóricos. Segundo a Organização Mundial de Saúde (OMS) [WHO 2018], adolescentes na faixa etária de 11 a 17 anos, em cada cinco quatro são sedentários. Nos adultos o sedentarismo está presente em 23\% dos indivíduos; já nos mais jovens, esse índice é de $81 \%$.

A OMS também afirma que a atividade física regular é fundamental, sendo excelente para a prevenção e tratamento de Doenças Crônicas não Transmissíveis (DCNTs). Essas enfermidades são responsáveis por $71 \%$ de todas as mortes no mundo, incluindo as mortes de 15 milhões de pessoas por ano entre 30 e 70 anos [OMS 2018].

Tendo em vista tamanha preocupação, a OMS lançou um plano de ação mundial sobre atividades físicas e saúde para 2018 a 2030, mostrando como os países podem 
reduzir a inatividade física em adultos e adolescentes em $15 \%$ durante todo esse período. Com isso, cresce a necessidade de incentivar pessoas a praticarem atividades físicas e a demanda por motivadores para mudança de hábitos em prol de uma vida mais saudável vem crescendo a cada dia.

Portanto, é importante ressaltar que com a crescente evolução das Tecnologias da Informação e Comunicação, o ser humano busca utilizar recursos tecnológicos afim de estabelecer formas mais eficazes para executar trabalhos complexos e cansativos quando desempenhados manualmente [Almondes et al. 2019]. Dentro deste contexto, os jogos digitais buscam proporcionar diversão, interação, conhecimento, desenvolvimento das habilidades motoras e, consequentemente, tornar as atividades rotineiras mais agradáveis de serem executadas.

Uma pesquisa da Universidade de Tóquio mostrou que o Pokémon $G O$ fez grande sucesso por usar mecânicas inovadoras em sua jogabilidade, contribuindo para diminuição de fatores como depressão, fobias sociais e o sedentarismo [Watanabe et al. 2017]. Mesmo de forma indireta, o jogo conseguiu que os usuários deixassem de ter um comportamento sedentário e praticassem esportes de forma involuntária. Essa mudança de comportamento foi possível devido as mecânicas incorporadas ao jogo, que por utilizarem Geolocalização e Realidade Aumentada faziam com que os jogadores saíssem de casa até o local onde os pokemons surgiam.

Seguindo a proposta apresentado no jogo Pokémon GO, The Wanderer foi desenvolvido fazendo uso de mecânicas que estimulam o interesse dos jogadores na mudança de comportamentos e hábitos que remetam ao sedentarismo. Porém, o seu diferencial está na possibilidade do jogador poder criar o seu próprio percurso de caminhada/corrida, podendo ser jogado em qualquer lugar, como corredores ou áreas de lazer. Outra vantagem é que não há necessidade de uma conexão com a internet para jogar, ao contrário do Pokémon $G O$, onde a conexão com a rede é imprescindível para o funcionamento do jogo.

\section{Trabalhos Relacionados}

O trabalho de [Fritsch 2015] traz um jogo do gênero MMORPG ${ }^{1}$ baseado em geolocalização e realidade aumentada para dispositivos móveis. Seu desenvolvimento foi baseado na metodologia de Bruno Munari e apoiado pelo artigo "MDA: A Formal Approach to Game Design and Game Research" [Hunicke et al. 2004].

O trabalho de [Marins et al. 2011] visa estimular a prática de atividades físicas por meio de um exergame ${ }^{2}$ de corrida avaliando a experiência do jogador como fator motivador para a mudanças de hábitos quanto a prática de atividades físicas.

O trabalho de [Macvean and Robertson 2012] busca incentivar crianças na faixa etária de 12 e 15 anos a praticar atividades físicas em níveis moderados e vigorosos. A proposta verifica e avalia a apreciação do jogo para atividades físicas, com base nas opiniões em relação ao jogo, a possibilidade de configurações de acordo com as necessidades físicas e, por fim, como ganhar ou perder influenciam na experiência do jogador.

\footnotetext{
${ }^{1}$ MMORPG: Massively Multiplayer Online Role-Playing Game Disponível em: https://www.techtudo.com.br/artigos/noticia/2012/08/o-que-e-mmorpg.html. Acesso em: 20 Jun. 2020

${ }^{2}$ Exergames são jogos digitais que requerem mais esforço físico ou movimentos do que o comportamento sedentário e incluem atividades de força, equilíbrio ou flexibilidade [Oh and Yang 2010].
} 
O presente trabalho busca incentivar o jogador a praticar caminhadas como atividade física principal. O uso de sensores para a detecção de passos força o jogador a se manter em movimento para que ele possa superar os vários desafios que aparecem em seu caminho e consiga chegar a novos cenários no jogo, avaliando também a sua experiencia.

\section{Metodologia do Trabalho}

A proposta foi desenvolvida em duas etapas. Na primeira foi feita uma investigação para identificar as diversas metodologias utilizadas para o desenvolvimento de um exergame. Através desta pesquisa foi possível determinar todo o potencial material que poderia ser utilizado na construção jogo, constatando-se que seria necessário achar uma forma de detectar ações feitas pelos jogadores e que algumas das soluções mais utilizadas no contexto de dispositivos móveis seria o uso de sensores.

Outra pesquisa foi realizada para caracterizar os padrões de movimentos que se encaixam na categoria de caminhada/corrida e as possíveis formas de rastrear e computar esses movimentos. Por essa razão, dentre os sensores que poderiam ser utilizados para a construção deste trabalho, o escolhido foi o acelerômetro por sua natureza especializada na detecção de aceleração feita sobre um determinado corpo.

Na segunda etapa, o foco foi definir quais temáticas se encaixariam dentro do contexto de um jogo voltado para promover o hábito da realização de atividades físicas. As temáticas abrangem todo o potencial conteúdo para compor o produto como, por exemplo, mecânicas do jogo, cenários e possíveis desafios. Optou-se pela utilização dos biomas brasileiros mais conhecidos, para que cada cenário pudesse ter diferenciais estéticos característicos e distintos.

Como cada bioma possui uma fauna e flora diferentes em sua grande maioria, eles apresentariam desafios diferentes para o jogador superar. Com essa diversidade o jogador seria entretido de variadas formas, contribuindo não apenas com seu engajamento na atividade física em questão, mas também no seu crescimento cultural acerca dos biomas brasileiros.

\subsection{Tecnologias Utilizadas}

A framework Unity $^{3}$ foi selecionada para a construção deste trabalho pela facilidade de uso e suporte a várias plataformas. A grande variedade de recursos disponíveis permitiu a implementação da proposta de forma eficaz e viabilizou a desenvolvimento de um sistema eficiente na deteç̧ão de caminhadas.

O sensor acelerômetro foi utilizado no sistema de detecção de caminhada por ser capaz de funcionar a partir de diversos efeitos físicos, como medir valores de aceleração de um determinado corpo [Toledo 2015]. O funcionamento desse sistema é baseado na tecnologia usada em óculos de realidade virtual (Virtual Reality ou VR), capazes de detectar movimentos do usuário. O sistema desenvolvido trabalha com intervalos de movimento, não sendo capaz de fazer uma classificação exata do tipo de movimento executado pelo jogador.

\footnotetext{
${ }^{3}$ Unity 3D. Disponível em: https://unity3d.com/pt. Acesso em: 20 Jun. 2020.
} 
Os softwares Blender ${ }^{4}$, Inkscape ${ }^{5}$ e Krita ${ }^{6}$ foram usados para criar os elementos gráficos de interfaces e modelos 3D. O software LMMS (Linux MultiMedia Studio) ${ }^{7}$ foi utilizado para a composição da trilha sonora e sons de interfaces presentes na aplicação.

O processo de avaliação e usabilidade de software foi feito utilizando a ferramenta Google Forms ${ }^{8}$. Os questionamentos aplicados levaram em consideração a metodologia proposta pelo modelo da escala SUS (System Usability Scale). O SUS possui um total de 10 perguntas, nas quais o usuário pode responder utilizando a escala Likert. Esta escala é usada para medir o grau de aceitação de uma pessoa sobre um determinado assunto.

\section{O jogo The Wanderer}

A proposta principal de The Wanderer (O Andarilho, em tradução literal) é ser um exergame focado em caminhada ou corrida. O jogo implementa características de games Role-Playing Game (RPG) e pode ser configurado para ser usado em dois idiomas (inglês e português). Segundo [Almondes et al. 2019] RPG são jogos onde o jogador vive um papel fictício de um personagem dentro do jogo. No mundo dos gamers o personagem adotado pelo usuário é conhecido como avatar.

\subsection{Menus}

A distribuição dos menus segue uma ordem lógica e linear, de maneira a facilitar a introdução de novos jogadores. Eles apresentam o jogo e suas funcionalidades conforme demonstra o fluxograma da Figura 1.

O jogador começa pela seleção do idioma a ser utilizado durante o percurso de sua atividade física. Caso seja a primeira vez, ele será levado para a apresentação/customização do avatar e tutorial do jogo. Caso contrário, será enviado para o Menu Principal. O tutorial apresenta algumas recomendações e em seguida são introduzidas as mecânicas presentes no contexto do jogo.

Ao finalizar o tutorial é apresentado o Menu Principal. Nele o jogador pode dar início à sua experiência escolhendo um dos modos de jogo (Trilha e Viagem). Dentro do Menu de Configurações, é possível realizar ajustes de idioma, volume ou customização do avatar. O Menu de Créditos exibe informações sobre os autores e, por fim, selecionando a opção Sair o jogo é finalizado. Na versão de testes, a opção Responder Questionário foi adicionada para redirecionar o jogador para o questionário de avaliação de usabilidade do software. As setas indicam a ordem de navegação entre os menus.

\subsection{Jogabilidade}

A mecânica de jogabilidade está dividida em duas categorias distintos: Trilha e Viagem.

- Trilha: Dentro desta categoria o jogador precisa andar até que seu percusso seja concluído, tomando cuidado com os obstáculos que surgem no caminho. O progresso aumenta conforme o jogador anda no mundo real.

\footnotetext{
${ }^{4}$ Blender. Disponível em: https://www.blender.org/. Acesso em: 20 Jun. 2020.

${ }^{5}$ Inkscape. Disponível em: https://inkscape.org/pt-br/. Acesso em: 20 Jun. 2020.

${ }^{6}$ Krita. Disponível em: https://krita.org/en/. Acesso em: 20 Jun. 2020.

${ }^{7}$ LMMS. Disponível em: https://github.com/LMMS/lmms. Acesso em: 20 Jun. 2020.

${ }^{8}$ Google Forms é uma ferramenta gratuita do Google LLC que permite a elaboração de questionários online. Disponível em: https://www.google.com/intl/pt-BR/forms/about/. Acesso em: 20 Jun. 2020.
} 


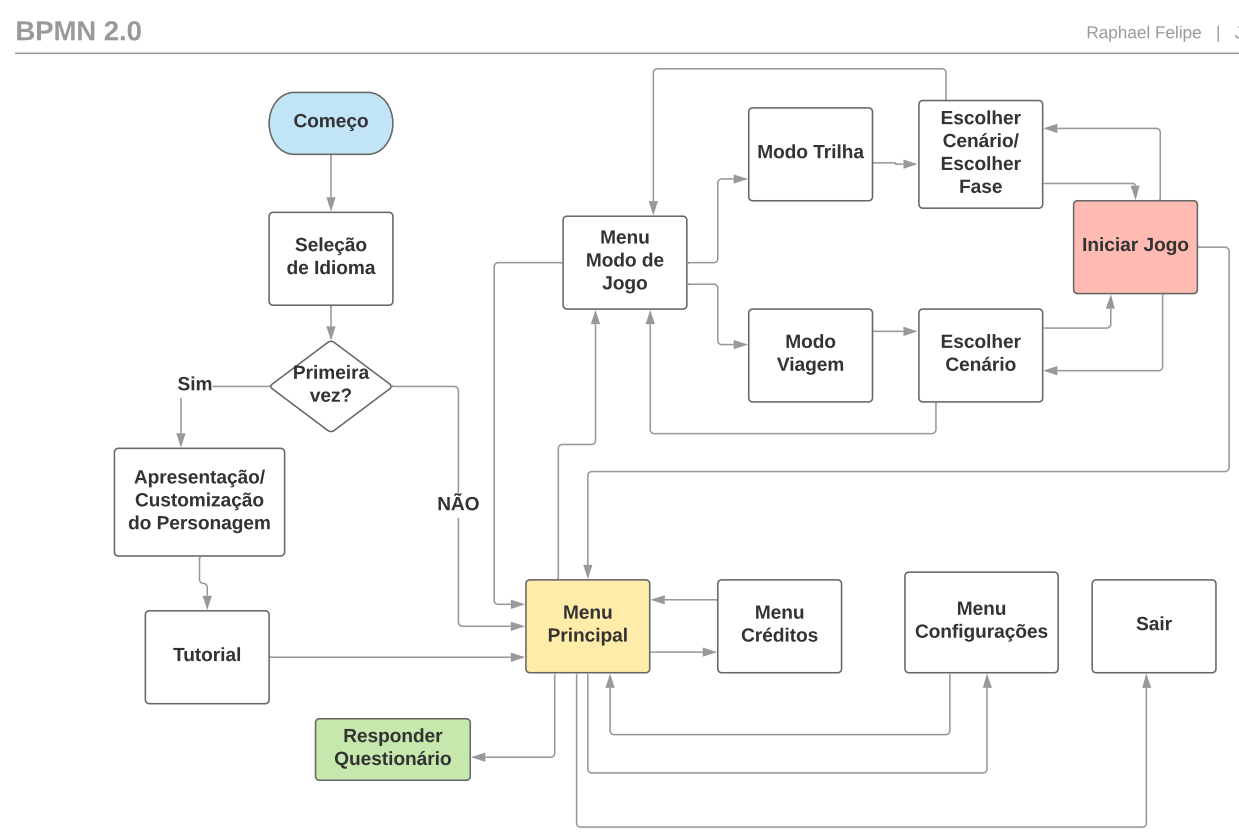

Figura 1. Fluxograma - Estrutura do jogo.

- Viagem: Nesta categoria o jogador não possui um tamanho de percusso definido. O tempo da atividade perdura enquanto o jogador tiver disponibilidade em executá-la.

Durante o percurso o jogador pode acumular pontos que poderão ser trocados por mais vidas para continuar no modo Trilha. Os pontos também são usados no Ranking dos jogadores. O sistema de recompensas variam em 2 tipos, podendo ser estéticas, agregando um novo visual ao avatar ou atributos extras que concedem vantagens durante o jogo. Podem ser também desafios, como novos cenários e tipos de obstáculos.

\subsection{Cenários}

The Wanderer possui ambientação inspirada em alguns biomas do Brasil como Amazônia, Mata Atlântica, Caatinga e Pantanal. A Figura 2 contém exemplos de alguns dos cenários presentes no jogo.

Elementos da fauna e da flora compõem cada um dos cenários de modo que venha a contribuir com a imersão e engajamento do jogador. Os cenários são liberados conforme o jogador progride na categoria de jogabilidade escolhida no início do jogo.

\subsection{Mecânicas de Jogo}

A principal mecânica de sustentação do The Wanderer é o sistema de caminhada. Através dela o jogador executa uma caminhada no mundo real, reproduzida pelo avatar no mundo virtual. Duas mecânicas auxiliares são utilizadas para incrementar a experiência do jogador: Quick Time Event(QTE) e Customização de Personagem(Custom Character ou $C C)$.

Um QTE (Figura 3.a) consiste em um momento onde o jogador deve realizar uma determinada ação em um instante específico. Eles surgem como obstáculos que jogador deve superar para continuar progredindo e necessitam de reflexos precisos. O sistema CC 


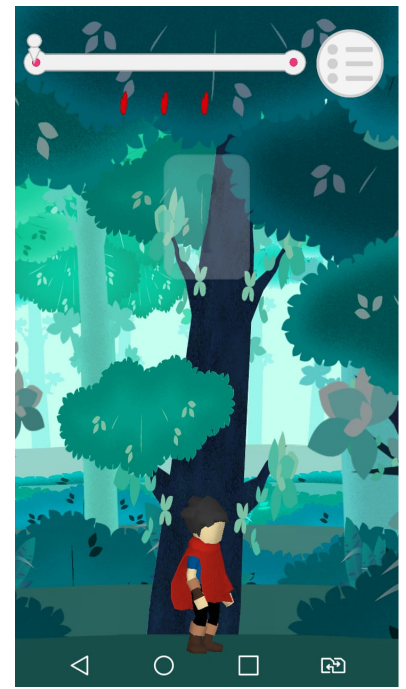

a)

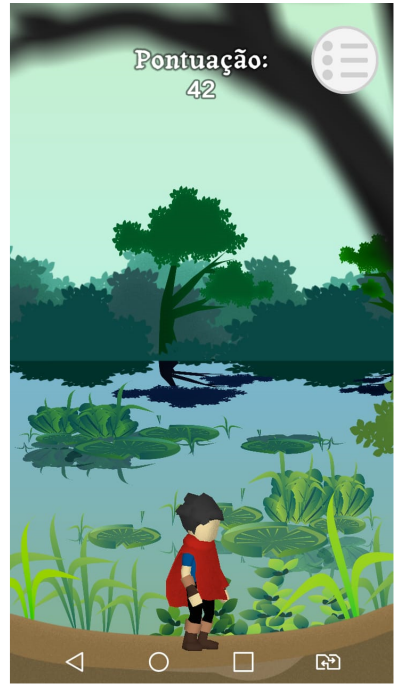

b)

Figura 2. Amostra de cenários: Floresta Amazônica (a) e Pantanal (b).

(Figura 3.b) permite criar um avatar virtual de acordo com as necessidades e gosto de cada jogador.

\section{Avaliação de Usabilidade do Software}

Um questionário foi disponibilizado a todos o jogadores que se dispuseram a participar dos testes de avaliação da aplicação mobile. O questionário não tinha caráter obrigatório e nenhuma informação pessoal foi requisitada dos participantes durante o processo de coleta de dados.

A metodologia utilizada para coleta de dados foi amparada na escala SUS, desenvolvida por Brooke em 1986. O instrumento SUS contém dez questões que visam medir a usabilidade de diversos produtos e serviços. É considerada robusta e versátil, de fácil entendimento, possui boa confiabilidade e suas referências ajudam na interpretação dos resultados [Padrini-Andrade et al. 2019]

As questões do questionário SUS foram graduadas em uma escala tipo Likert, com valores variando de 1 (um) a 5 (cinco). A escala foi classificada como segue: "discordo completamente (1)", "discordo (2)", "neutro (3)", "concordo (4)" e "concordo completamente (5)" [Lucian and Dornelas 2015].

\subsection{Cálculo do Questionário}

Para calcular a pontuação, primeiro é somado os pontos de cada item que contribui em uma escala de 1 a 5. Para os itens 1, 3, 5, 7 e 9, a pontuação individual é a nota recebida menos 1. Para os itens 2, 4, 6, 8 e 10, se atribui 5 menos a nota recebida. Multiplica-se a soma de todos os pontos por 2,5 e assim é obtido o valor total do SUS, conforme o exemplo exibido na Figura 4 [Brooke 1996].

A pontuação do SUS varia entre 0 e 100 . A classificação do sistema avaliado é feito de acordo com a pontuação final: menor que 20,5 (pior imaginável); 21 a 38,5 (pobre); 39 a 52,5 (mediano); 53 a 73,5 (bom); 74 a 85,5 (excelente); e 86 a 100 (melhor imaginável) [Martins et al. 2015]. 


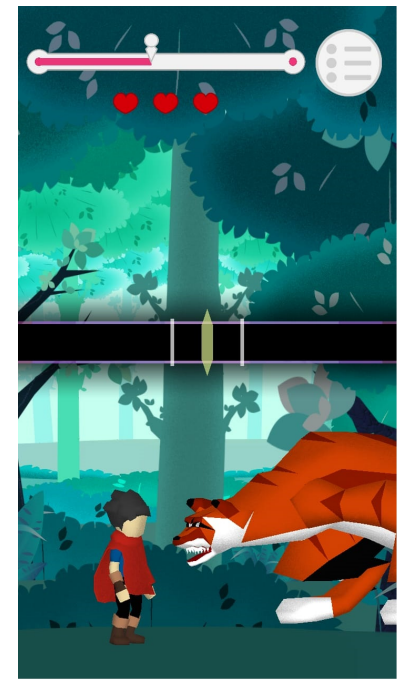

a)

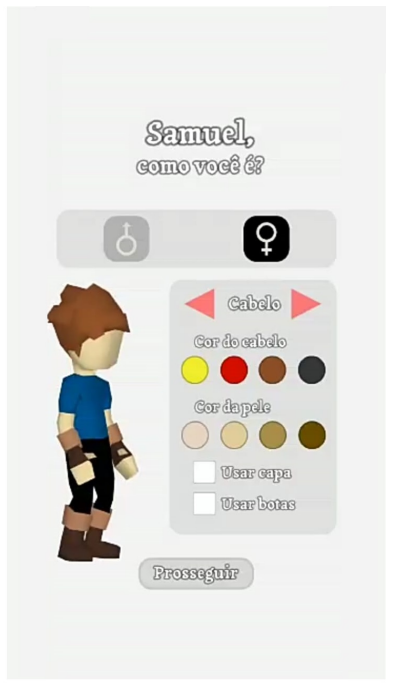

b)

Figura 3. Exemplo de Quick Time Event(a) e Tela de Customização de Personagem(b).

\begin{tabular}{|c|c|c|c|c|c|c|c|c|c|}
\hline q1 & q2 & q3 & q4 & q5 & q6 & q7 & q8 & q9 & q10 \\
\hline 5 & 2 & 5 & 2 & 5 & 1 & 4 & 1 & 5 & 1 \\
\hline
\end{tabular}

Questôes ímpares: $(5-1)+(5-1)+(5-1)+(4-1)+(5-1)=4+4+4+3+4=19$

Questôes pares: $(5-2)+(5-2)+(5-1)+(5-1)+(5-1)=3+3+4+4+4=18$

Expressão: soma das questôes $\times 2,5=37 \times 2,5=92,5$

Classificação: melhor imaginável

Figura 4. Exemplo de respostas em um questionário SUS. Fonte: [Padrini-Andrade et al. 2019].

\subsection{Resultados}

O jogo The Wanderer leva o jogador a se manter em movimento a todo momento, sendo que a caminhada é o único modo de poder progredir, ou seja, para que possa concluir cada cenário e superar cada desafio proposto pelo jogo, o jogador não deve parar de andar. Tendo isso em mente, é necessário que o software possa facilitar a compreensão do jogador quanto ao objetivo do jogo e auxilia-lo durante todo o processo.

Participaram do processo de jogabilidade e avaliação do software, 13 pessoas. Segundo Nielsen [Jakob 2000], com 5 participantes já é possível encontrar $80 \%$ dos problemas de uma interface. Depois disso os problemas começam a se repetir e o aprendizado diminui. Em outras palavras, uma amostra com 5 participantes tem um bom custo benefício. Com base nisso, o número de participantes que prontificaram-se a responder ao questionário foi considerado satisfatório.

A média do SUS(score) é 68 pontos que corresponde a $50 \%$ de aceitação [Ribeiro 2017]. Uma boa pontuação está acima de 80,3 pontos. Produtos que apresentam esta pontuação estão mais propícios de indicação de uso após o teste. Pontuações inferiores a 51 indicam que as interfaces possuem sérios problemas a serem solucionados [Sauro 2011]. A Figura 5 apresenta o resultado do questionário da avaliação de usabili- 
dade do software The Wanderer.

\begin{tabular}{|c|c|c|c|c|c|c|c|}
\hline Perguntas & $\begin{array}{c}\text { Discordo } \\
\text { Completamente }\end{array}$ & Discordo & Neutro & Concordo & $\begin{array}{c}\text { Concordo } \\
\text { Completamente }\end{array}$ & Participantes & Pontuação Individual \\
\hline A proposta apresentada é muito interessante. & $0,00 \%$ & $0,00 \%$ & $7,70 \%$ & $7,70 \%$ & $84,60 \%$ & Participante 01 & 87,5 \\
\hline \multirow{2}{*}{$\begin{array}{l}\text { NÃO jogaria algo relacionado com a temática } \\
\text { desta proposta. }\end{array}$} & \multirow{2}{*}{$61,50 \%$} & \multirow[b]{2}{*}{$30,80 \%$} & \multirow[b]{2}{*}{$0,00 \%$} & \multirow[b]{2}{*}{$7,70 \%$} & \multirow[b]{2}{*}{$0,00 \%$} & Participante 02 & 100 \\
\hline & & & & & & Participante 03 & 87,5 \\
\hline \multirow{2}{*}{$\begin{array}{l}\text { O jogo possui elementos consistentes e são } \\
\text { fáceis de serem compreendidos. }\end{array}$} & \multirow{2}{*}{$0,00 \%$} & \multirow{2}{*}{$0,00 \%$} & \multirow{2}{*}{$0,00 \%$} & \multirow{2}{*}{$30,80 \%$} & \multirow{2}{*}{$69,20 \%$} & Participante 04 & 100 \\
\hline & & & & & & Participante 05 & 97,5 \\
\hline $\begin{array}{l}\text { O jogo possui elementos inconsistentes e são } \\
\text { difíceis de serem compreendidos. }\end{array}$ & $61,50 \%$ & $30,80 \%$ & $0,00 \%$ & $7,70 \%$ & $0,00 \%$ & Participante 06 & 90 \\
\hline \multirow{3}{*}{$\begin{array}{l}\text { O jogo funcionou completamente bem e não } \\
\text { apresentou problemas que comprometessem } \\
\text { minha experiência. }\end{array}$} & \multirow{3}{*}{$0,00 \%$} & \multirow{3}{*}{$0,00 \%$} & \multirow{3}{*}{$0,00 \%$} & \multirow{3}{*}{$46,20 \%$} & \multirow{3}{*}{$53,80 \%$} & Participante 07 & 100 \\
\hline & & & & & & Participante 08 & 80 \\
\hline & & & & & & Participante 09 & 77,5 \\
\hline $\begin{array}{l}\text { O jogo apresentou muitos problemas durante o } \\
\text { uso que comprometeram minha experiência. }\end{array}$ & $69,20 \%$ & $23,10 \%$ & $0,00 \%$ & $0,00 \%$ & $7,70 \%$ & Participante 10 & 90 \\
\hline O jogo possui telas muito intuitivas e bem & $0,00 \%$ & $0,00 \%$ & $0,00 \%$ & $15,40 \%$ & $84,60 \%$ & Participante 11 & 87,5 \\
\hline integradas entre si. & & & & $10,+00$ & $84,60 \%$ & Participante 12 & 92,5 \\
\hline \multirow{2}{*}{$\begin{array}{l}\text { O jogo possui telas muito complexas e } \\
\text { mal integradas. }\end{array}$} & \multirow{2}{*}{$100,00 \%$} & \multirow{2}{*}{$0,00 \%$} & \multirow{2}{*}{$0,00 \%$} & \multirow{2}{*}{$0,00 \%$} & \multirow{2}{*}{$0,00 \%$} & Participante 13 & 87,5 \\
\hline & & & & & & \multicolumn{2}{|c|}{ Pontuação Geral } \\
\hline O jogo e multo alvertaoo e bontlo. & $0,00 \%$ & $0,00 \%$ & $23,10 \%$ & $38,50 \%$ & $38,50 \%$ & & 90,6 \\
\hline O jogo é muito chato, com péssimos gráficos. & $84,60 \%$ & $15,40 \%$ & $0,00 \%$ & $0,00 \%$ & $0,00 \%$ & & \\
\hline
\end{tabular}

Figura 5. Resultado do questionário da avaliação de usabilidade do software The Wanderer.

Na Figura 5 são demonstradas as avaliações de cada participante e a pontuação geral do software. É possível perceber que a atribuição individual feita por cada participante foi de no mínimo 80 pontos (Figura 5.a). Segundo [Sauro 2011] este valor está muito próximo de 80,3 que é considerado um ótimo score. A avaliação geral de usabilidade do software(score) foi de 90,6 pontos (Figura 5.b), mostrando que o trabalho é promissor ao propor a prática de atividades físicas ao usuário final.

\section{Conclusão}

Jogos digitais já se provaram eficientes na maioria dos contextos em que foram inseridos. O software proposto neste trabalho busca comprovar a sua capacidade em levar o jogador a prática de atividades físicas, utilizando-se de vários desafios que devem ser superados ao longo de cada fase de jogo, de uma forma lúdica e interessante.

Através dos resultados obtidos nos questionários de teste de usabilidade de software, The Wanderer obteve uma avaliação média de usabilidade de 90,6 pontos e uma média de aceitação de $94,62 \%$, de acordo com o modelo proposto pela escala SUS. Estes resultados apontam sua viabilidade no incentivo de práticas de atividades físicas, mostrando-se ser uma ferramenta capaz de auxiliar no combate ao sedentarismo.

Para trabalhos futuros pretende-se: (a) aperfeiçoar o sistema de captação de caminhadas, usando aprendizado de máquina para um classificação mais precisa e eficiente do tipo de atividade que o jogador esta executando e; (b) disponibilizar o jogo em plataformas de mídias digitais para maior acessibilidade ao público.

\section{Referências}

Almondes, J., Imperes Filho, F., dos Santos, A. R. F., de Leal, I., and Machado, V. (2019). Schoolg: Uma ferramenta computacional voltada para o auxílio no ensino de geografia no 5o. ano do ensino fundamenta. In Brazilian Symposium on Computers in Education (Simpósio Brasileiro de Informática na Educação-SBIE), volume 30, page 783.

Brooke, J. (1996). Sus: a “quick and dirty'usability. Usability evaluation in industry, page 189 . 
IX Congresso Brasileiro de Informática na Educação (CBIE 2020)

Anais do XXXI Simpósio Brasileiro de Informática na Educação (SBIE 2020)

Fritsch, G. O. (2015). Jogo digital baseado em geolocalização e realidade aumentada.

Hunicke, R., LeBlanc, M., and Zubek, R. (2004). Mda: A formal approach to game design and game research. In Proceedings of the AAAI Workshop on Challenges in Game AI, volume 4, page 1722.

Jakob, N. (2000). Why you only need to test with 5 users. Nielsen Norman Group, Nielsen.

Lucian, R. and Dornelas, J. S. (2015). Mensuração de atitude: Proposição de um protocolo de elaboração de escalas. RAC-Revista de Administração Contemporânea, 19(2):157177.

Macvean, A. and Robertson, J. (2012). ifitquest: a school based study of a mobile location-aware exergame for adolescents. pages 359-368.

Marins, D. R., de OD Justo, M., Xexeo, G. B., de AM Chaves, B., et al. (2011). Smartrabbit: A mobile exergame using geolocation. pages 232-240.

Martins, A. I., Rosa, A. F., Queirós, A., Silva, A., and Rocha, N. P. (2015). European portuguese validation of the system usability scale (sus). Procedia Computer Science, 67:293-300.

Oh, Y. and Yang, S. (2010). Defining exergames \& exergaming.

OMS (2018). Oms lança plano de ação global sobre atividade física para reduzir comportamento sedentário e promover a saúde.

Padrini-Andrade, L., Balda, R. d. C. X., Areco, K. C. N., Bandiera-Paiva, P., Nunes, M. d. V., Marba, S. T. M., Carvalho, W. B. d., Rugolo, L. M. S. d. S., Almeida, J. H. C. d., Procianoy, R. S., et al. (2019). Avaliação da usabilidade de um sistema de informação em saúde neonatal segundo a percepção do usuário. Revista Paulista de Pediatria, 37(1):90-96.

Ribeiro, R. O. (2017). Usabilidade e aplicativos mobile de bibliotecas. B.S. thesis.

Sauro, J. (2011). Does prior experience affect perceptions of usability. Retrieved December, 20:I1.

Toledo, L. (2015). Fusão de sensores para localização espacial com filtro em sistema markoviano.

Watanabe, K., Kawakami, N., Imamura, K., Inoue, A., Shimazu, A., Yoshikawa, T., Hiro, H., Asai, Y., Odagiri, Y., Yoshikawa, E., et al. (2017). Pokémon go and psychological distress, physical complaints, and work performance among adult workers: a retrospective cohort study. Scientific reports, 7(1):10758.

WHO (2018). Global action plan on physical activity 2018-2030: more active people for a healthier world. World Health Organization. 